\title{
Successful reduction of overexposure in patients with rheumatoid arthritis with high serum adalimumab concentrations: an open-label, non-inferiority, randomised clinical trial
}

\author{
Merel J I'Ami, ${ }^{1}$ Charlotte LM Krieckaert, ${ }^{1}$ Michael T Nurmohamed, ${ }^{1,2}$ \\ Ronald F van Vollenhoven, ${ }^{1,2,3}$ Theo Rispens, ${ }^{4}$ Maarten Boers, ${ }^{2,5}$ Gerrit Jan Wolbink ${ }^{1,4}$
}

\begin{abstract}
Handling editor Tore K Kvien
- Additional material is published online only. To view please visit the journal online (http://dx.doi.org/10.1136/ annrheumdis-2017-211781)
\end{abstract}

${ }^{1}$ Rheumatology, Amsterdam Rheumatology and Immunology Center, Location Reade, Amsterdam, Netherlands ${ }^{2}$ Rheumatology, Amsterdam Rheumatology and Immunology Center, Location VU University Medical Center, Amsterdam, Netherlands

${ }^{3}$ Rheumatology, Amsterdam Rheumatology and Immunology Center, Location Amsterdam Medical Center, Amsterdam, Netherlands

${ }^{4}$ Immunopathology, Sanquin

Research and Landsteiner Laboratory, Amsterdam,

Netherlands

${ }^{5}$ Epidemiology \& Biostatistics, VU University Medical Center, Amsterdam, Netherlands

Correspondence to Merel J I'Ami, Amsterdam Rheumatology and Immunology Center, Amsterdam 1056 AB, The Netherlands: m.lami@reade.nl

Received 12 May 2017 Revised 13 July 2017 Accepted 8 September 2017 Published Online First 22 September 2017

- http://dx.doi.org/10.1136/ annrheumdis-2017-212376

Check for updates

To cite: I'Ami MJ Krieckaert CLM,

Nurmohamed MT,

et al. Ann Rheum Dis

2018;77:484-487.

\section{ABSTRACT}

Objective High adalimumab serum concentrations do not result in better response in patients with rheumatoid arthritis (RA), suggesting overexposure. We investigated whether patients with adalimumab concentrations $>8 \mu \mathrm{g} / \mathrm{mL}$ can prolong their dosing interval by $50 \%$ without a clinically relevant change in disease activity. Methods Consecutive patients with RA, treated with adalimumab $40 \mathrm{mg}$ every other week for at least 28 weeks, were approached for this randomised, open-label, non-inferiority trial. Patients with adalimumab trough concentrations $>8 \mu \mathrm{g} / \mathrm{mL}$ were randomly $(1: 1)$ assigned to dose-interval prolongation of once every 3 weeks or continuation of every other week. Primary outcome was the change in disease activity score of 28 joints ( $\triangle$ DAS28-ESR) after 28 weeks, with a non-inferiority margin of 0.6 points.

Results In total, 147 patients were screened. Fiftyfive patients had concentrations $>8 \mu \mathrm{g} / \mathrm{mL}$ and were randomised. Mean $\triangle D A S 28$ after 28 weeks was $-0.14 \pm$ SD 0.61 in the prolongation group and $0.30 \pm 0.52$ in the continuation group. Mean difference was significantly in favour of the prolongation group: 0.44 ( $95 \% \mathrm{Cl} 0.12$ to $0.76, p=0.01)$.

Conclusions Adalimumab-treated patients with RA with trough concentrations $>8 \mu \mathrm{g} / \mathrm{mL}$ can prolong their standard dosing interval to once every 3 weeks without loss of disease control.

Trial registration number NTR3509; Results.

\section{INTRODUCTION}

Biologic disease-modifying anti-rheumatic drugs (bDMARDs) have become important in the treatment of rheumatoid arthritis (RA) and improved prognosis. ${ }^{1} 2$ Adalimumab, a monoclonal antibody targeting tumour necrosis factor (TNF), is frequently prescribed in RA. Several factors (such as immunogenicity) strongly affect its pharmacokinetics, resulting in a wide range of adalimumab concentrations at the approved dose. ${ }^{3-7}$ Higher adalimumab concentrations result in better clinical response, but the curve plateaus around $5 \mu \mathrm{g} / \mathrm{mL}$. $^{56}$ Thus, patients with steady-state trough concentrations above $5 \mu \mathrm{g} / \mathrm{mL}$ are most likely overexposed.

Identification of and acting on overexposure will reduce drug costs and possibly risk of adverse events. ${ }^{89}$ We investigated whether we could prolong the interval by $50 \%$ in patients with RA with adalimumab trough concentrations of at least $8 \mu \mathrm{g} / \mathrm{mL}$ without affecting disease control.

\section{METHODS}

\section{Study design, patients and intervention}

This 28-week, open-label, randomised, parallel-group, non-inferiority trial was performed in the Amsterdam Rheumatology and immunology Center, location Reade, The Netherlands. A full description of the methods is provided in the online supplementary appendix. Briefly, adalimumab serum trough concentrations were determined in patients with RA (American College of Rheumatology 1987 criteria $^{10}$ ) who had been treated with adalimumab $40 \mathrm{mg}$ every other week for at least 28 weeks and had no indication for adjustment of adalimumab treatment, discontinuation or a scheduled surgery in the next 6 months. Patients with concentrations above $8 \mu \mathrm{g} / \mathrm{mL}$ were randomly assigned (1:1) to prolongation of the interval to $40 \mathrm{mg}$ once every 3 weeks (prolongation group) or to continuation of the standard interval of every other week (continuation group). Both groups were followed for 28 weeks to ensure new steady state of adalimumab concentrations. The treating rheumatologist could change co-medication during follow-up. Given the linear clearance of adalimumab, ${ }^{5}$ the cut-off of $8 \mu \mathrm{g} / \mathrm{mL}$ was chosen to maintain concentrations above $5 \mu \mathrm{g} / \mathrm{mL}$ after interval prolongation to once every 3 weeks. The protocol was approved by the Slotervaart Hospital and Reade Medical Research Ethics Committee and was registered at the Dutch Trial Register (www.trialregister.nl) with trial number NTR3509. Patients gave written informed consent.

\section{Outcomes}

The primary endpoint was the change in disease activity score of 28 joints ( $\triangle \mathrm{DAS} 28$-ESR) after 28 weeks. A clinically relevant $\triangle \mathrm{DAS} 28$ was defined as 0.6 points or more. ${ }^{11}$ Other outcomes included the change in clinical and simplified disease activity score (respectively CDAI and SDAI) and adalimumab concentration after week 28. Clinical and laboratory assessments were scheduled at baseline, 12 and 28 weeks (visits 1, 2 and 3, respectively) and adverse events were monitored during follow-up. 
Adalimumab concentration $[\mu \mathrm{g} / \mathrm{mL}]$

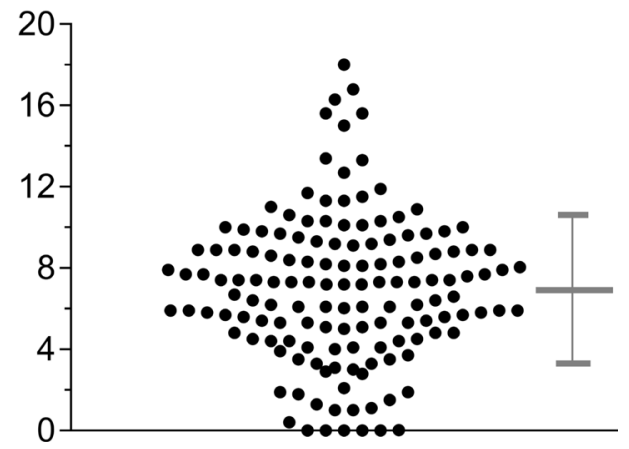

Figure 1 Wide variation exists in adalimumab concentrations ( $\mu \mathrm{g} /$ $\mathrm{mL}$ ) of screened patients. Each dot represents one patient. Patients with adalimumab concentration above $8 \mu \mathrm{g} / \mathrm{mL}$ were eligible to participate in the study. Mean and SD are shown in lines on the right.

Adalimumab serum trough concentrations were measured by ELISA previously described in detail. ${ }^{6}$

\section{Statistical analysis}

The sample size calculation was based on the non-inferiority principle, to exclude a difference in $\triangle \mathrm{DAS} 28 \geq 0.6$ to the detriment of the prolongation group with a one-sided $95 \%$ confidence boundary and a power of $80 \%$, resulting in a sample size of 102 patients. Given an anticipated dropout rate of 10\%, 56 patients were calculated per group. In case of a difference in favour of the prolongation group, this difference would be tested for significance with a two-sided test (alpha 5\%). T-tests were used for continuous variables and Fisher's exact tests for proportions. Unless otherwise indicated, analyses were by intention to treat.

\section{RESULTS \\ Patients}

A total of 238 patients were assessed for eligibility for the study; 86 patients refused to participate and 5 were excluded (trial profile in online supplementary appendix figure S1). Out of 147 screened patients, 108 had an adalimumab concentration above $5 \mu \mathrm{g} / \mathrm{mL}(73 \%)$ and 55 patients (37\%) above $8 \mu \mathrm{g} / \mathrm{mL}$ (figure 1); the latter were randomised. Fifty-four patients were included for analyses and 53 completed follow-up (baseline characteristics; table 1). Because of frequently recurring infections, 1 patient prolonged the dosing interval to every 3 weeks (decided by the treating rheumatologist) after 18 weeks in the continuation group. Methotrexate dose was changed by the treating rheumatologist in 6 patients and an intra-articular injection was given to 2 patients in the continuation group (online supplementary appendix; results). Although the inclusion period was extended with 2 years, the inclusion had to be stopped without reaching the target sample size. The main reason was a change in the institution preference policy for bDMARDs decreasing the number of eligible patients. The moment the study stopped, study staff had no knowledge of the accumulating study results.

Table 1 Demographics, previous and concomitant therapies, and disease status

\begin{tabular}{|c|c|c|c|c|c|c|c|c|}
\hline \multirow{2}{*}{ Demographics } & \multicolumn{4}{|c|}{ Interval prolongation $(n=27)$} & \multicolumn{4}{|c|}{ Continuation $(n=27)$} \\
\hline & \multicolumn{2}{|c|}{ Baseline } & \multicolumn{2}{|c|}{ Week 28} & \multicolumn{2}{|c|}{ Baseline } & \multicolumn{2}{|c|}{ Week 28} \\
\hline Age, mean \pm SD years & \multicolumn{2}{|c|}{$60 \pm 10$} & \multicolumn{2}{|l|}{-} & \multicolumn{2}{|c|}{$58 \pm 13$} & \multicolumn{2}{|l|}{-} \\
\hline Female, $\mathrm{n}(\%)$ & & (93) & \multicolumn{2}{|l|}{-} & \multicolumn{2}{|c|}{$26 \quad(96)$} & \multicolumn{2}{|l|}{-} \\
\hline $\mathrm{BMI}$, mean \pm SD & \multicolumn{2}{|c|}{$24.8 \pm 5.0$} & - & & 23.8 & $=4.3$ & - & \\
\hline \multicolumn{9}{|l|}{ Previous therapies } \\
\hline Prior DMARDs, median (IQR) & 3 & $(2-3)$ & \multicolumn{2}{|l|}{-} & 2 & $(2-3)$ & \multicolumn{2}{|l|}{-} \\
\hline Prior biologic, $\mathrm{n}(\%)$ & 4 & $(15)$ & \multicolumn{2}{|l|}{-} & 3 & $(11)$ & \multicolumn{2}{|l|}{-} \\
\hline Adalimumab treatment, median (IQR) years & 6.0 & $(2.9-8.0)$ & \multicolumn{2}{|l|}{-} & 5.5 & $(1.8-8.3)$ & \multicolumn{2}{|l|}{-} \\
\hline \multicolumn{9}{|l|}{ Concomitant therapies } \\
\hline MTX use, $n(\%)$ & 26 & (96) & 25 & (93) & 25 & (93) & 25 & (93) \\
\hline MTX dosage, median (IQR) mg/week & 20 & $(15-21)$ & 15 & $(11-20)$ & 15 & $(10-20)$ & 15 & $(10-20)$ \\
\hline Prednisone use, n (\%) & 4 & (15) & 4 & $(15)$ & 1 & (4) & 0 & $(0)$ \\
\hline \multicolumn{9}{|l|}{ Disease status } \\
\hline Disease duration, median (IQR) years & 11 & $(8-18)$ & \multicolumn{2}{|l|}{-} & 11 & $(6-19)$ & \multicolumn{2}{|l|}{-} \\
\hline ACPA positive, $n(\%)$ & 18 & $(67)$ & \multicolumn{2}{|l|}{-} & 21 & (78) & \multicolumn{2}{|l|}{-} \\
\hline IgM-RF positive, $n(\%)$ & 20 & (74) & \multicolumn{2}{|l|}{-} & 20 & (74) & \multicolumn{2}{|l|}{-} \\
\hline Erosive disease, $\mathrm{n}(\%)$ & 17 & (63) & \multicolumn{2}{|l|}{-} & 17 & $(63)$ & - & \\
\hline Primary outcome: $\mathrm{DAS} 28-\mathrm{ESR}$, mean \pm SD & 2.0 & \pm 0.8 & & 0.7 & 1.6 & 0.7 & 2.0 & 0.9 \\
\hline Patient's pain, median (IQR) mm & 15 & $(3-38)$ & 17 & $(4-34)$ & 10 & $(6-21)$ & 13 & $(9-22)$ \\
\hline Patient's global, median (IQR) mm & 15 & $(3-37)$ & 16 & $(4-37)$ & 15 & $(11-25)$ & 19 & $(8-35)$ \\
\hline Physician's global, median (IQR) mm & 4 & $(3-14)$ & 9 & $(2-21)$ & 12 & $(5-17)$ & 15 & $(7-26)$ \\
\hline Swollen joint count, median (IQR) & 0 & $(0-0)$ & 0 & $(0-0)$ & 0 & $(0-0)$ & 0 & $(0-0)$ \\
\hline Tender joint count, median (IQR) & 0 & $(0-0)$ & 0 & $(0-0)$ & 0 & $(0-0)$ & 0 & $(0-0)$ \\
\hline ESR, median (IQR) mm/hour & 9 & $(5-18)$ & 7 & $(5-18)$ & 5 & $(3-11)$ & 7 & $(4-14)$ \\
\hline CRP, median (IQR) mg/L & 1 & $(1-3)$ & 1 & $(1-2)$ & 1 & $(1-3)$ & 1 & $(1-2)$ \\
\hline HAQ, median (IQR) & 0.4 & $(0.1-1.0)$ & 0.5 & $(0.1-0.8)$ & 0.5 & $(0.1-1.3)$ & 0.6 & $(0.1-1.1)$ \\
\hline
\end{tabular}

ACPA, anticitrullinated protein antibody; BMI, body mass index; CRP, C-reactive protein; DAS28, 28 joints disease activity score; DMARDs, disease-modifying anti-rheumatic drugs; ESR, erythrocyte sedimentation rate; HAQ, Health Assessment Questionnaire; IgM-RF, IgM rheumatoid factor; MTX, methotrexate; SD, standard deviation. 


\section{Disease activity}

Mean DAS28 improved minimally in the prolongation group $(\triangle \mathrm{DAS} 28-0.14 \pm$ SD $0.61, \mathrm{n}=27)$ and deteriorated somewhat in the continuation group ( $\triangle \mathrm{DAS} 28 \quad 0.30 \pm 0.52, \mathrm{n}=24$; figure 2 ). The difference in $\triangle \mathrm{DAS} 28$ at 28 weeks was 0.44 in favour of the prolongation group $(95 \% \mathrm{CI}-0.76$ to $-0.12 ; \mathrm{p}=0.01)$. Seven patients $(26 \%)$ in the prolongation group versus 10 patients $(37 \%)$ in the continuation group had an increase in DAS28 $\geq 0.6$ points after 28 weeks $(\mathrm{p}=0.56)$. Two out of these 7 preferred to return to the standard dose. Four other patients also preferred to return to the standard dose after interval prolongation (online supplementary appendix table S1). Results were similar for SDAI and CDAI (online supplementary appendix; results).

\section{Adalimumab concentrations}

In the prolongation group, mean adalimumab concentration decreased from $10.6 \pm 2.5 \mu \mathrm{g} / \mathrm{mL}$ to $6.6 \pm 2.0 \mu \mathrm{g} / \mathrm{mL} \quad(\mathrm{n}=26)$ at week 28 (figure 2). In seven patients, the concentration decreased below $5 \mu \mathrm{g} / \mathrm{mL}$ during 28 -week follow-up. One of these 7 patients had an increase in DAS2 $8 \geq 0.6$ and returned to standard dose. In the continuation group, the concentration decreased slightly from $10.4 \pm 2.4 \mu \mathrm{g} / \mathrm{mL}$ to $9.3 \pm 3.0 \mu \mathrm{g} / \mathrm{mL}$ $(n=23)$; mean difference between the groups at week 28 was $2.6 \mu \mathrm{g} / \mathrm{mL}(95 \%$ CI 1.2 to $4.1 ; \mathrm{p}=0.001)$.

\section{Safety and adverse events}

Fourteen patients reported an adverse event in the continuation group and 2 patients in the prolongation group (online supplementary appendix table S2). No serious adverse events were reported.

\section{DISCUSSION}

This study shows that adalimumab-treated RA patients with trough serum concentrations above $8 \mu \mathrm{g} / \mathrm{mL}$ can prolong their
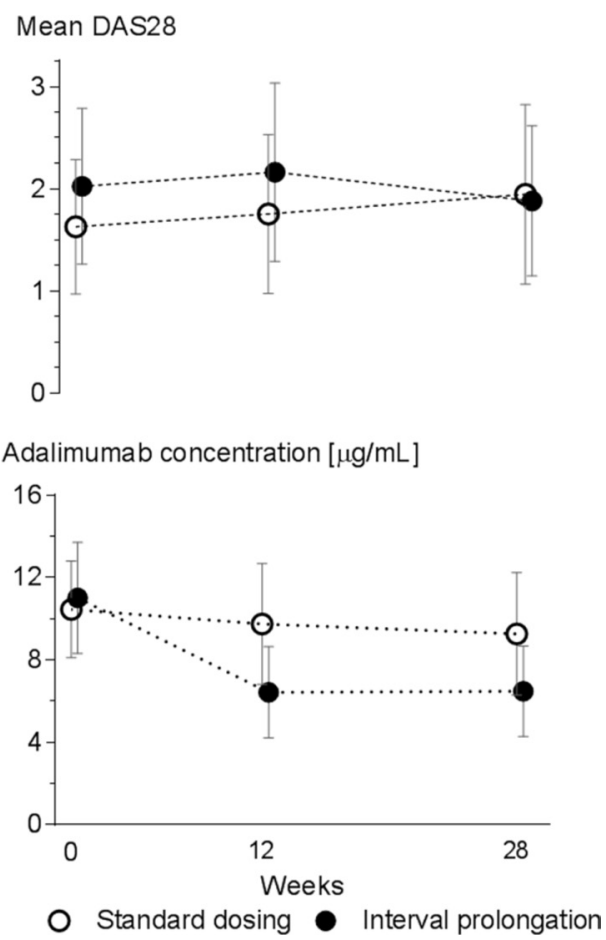

Figure 2 Mean (SD) disease activity score of 28 joints (DAS28) and mean (SD) adalimumab concentration $(\mu \mathrm{g} / \mathrm{mL})$ after interval prolongation or continuation of the standard dose. dosing interval to once every 3 weeks without an increase in disease activity. Considering mean change in disease activity, the prolongation group did well. Correspondingly, in most patients the adalimumab concentration remained above $5 \mu \mathrm{g} / \mathrm{mL}$, previously shown to be the concentration needed to block TNF. ${ }^{6}$ In the few patients where adalimumab concentrations decreased slightly below this level, it had no clinical consequences in the 28 weeks thereafter.

We observed an adalimumab concentration above $5 \mu \mathrm{g} / \mathrm{mL}$ in $73 \%$ of the patients. Avoiding overexposure is important in view of cost and possibly the risk of adverse events. ${ }^{89}$ Overexposure as a result of high adalimumab concentrations is independent of disease activity since the cut-off of $5 \mu \mathrm{g} / \mathrm{mL}$ is observed in patients with active disease. For many patients in remission, concentrations lower than $5 \mu \mathrm{g} / \mathrm{mL}$ might be sufficient to remain in that state. ${ }^{12-17}$

This study has some limitations. Adalimumab treatment for at least 28 weeks was required for participation, but the median treatment duration with adalimumab was 5.5 years. As the concentration-response relationship is based on 28 weeks of treatment, we do expect interval prolongation to be possible shortly after initiation of adalimumab treatment. Although disease activity was no inclusion criterion, the study population comprised mainly patients in minimal disease activity state or remission. As noted above, we expect that a proportion of these patients could prolong the dosing interval even further. DAS28 was missing in 3 out of 6 patients at the time they returned to standard dose. In the scenario that these patients all had an increase in DAS28 $\geq 0.6$, a total of 10 patients instead of 7 would have had a clinically relevant increase in disease activity. However, 10 patients in the continuation group also had an increase in DAS28 $\geq 0.6$, suggesting such increases are part of the natural fluctuation in DAS28 during standard-dose treatment. ${ }^{18}$ In addition, expectation bias is suggested by the fact that some patients elected to go back to standard dosing even though DAS28 had not increased to a relevant degree. Although DAS28 may be insensitive to relevant changes in the low disease activity range, we saw similar effects in SDAI and CDAI. Nevertheless, more sensitive outcomes might be necessary in future studies to detect flares. Another potential limitation is the failure to include the required number of patients. All patients treated with adalimumab at our centre were approached for the study. However, fewer patients than expected were treated with adalimumab and around one-third refused to participate. Fear for relapse after interval prolongation was an often heard reason. As it happened, post hoc power was more than sufficient to document non-inferiority because change in DAS28 was statistically in favour to the prolongation group.

Drug costs can be reduced by $33 \%$, that is, $€ 5000$ yearly per patient based on the cost of standard-dose adalimumab treatment in the Netherlands. Beyond the scope of our study, similar concentration-response relationships have been published for adalimumab treatment in psoriatic arthritis and psoriasis, suggesting overexposure may be a feature in these diseases as well. ${ }^{1920}$ Moreover, this study demonstrates the potential benefit of therapeutic drug monitoring.

In conclusion, this study shows that patients with RA on adalimumab with serum trough concentrations above $8 \mu \mathrm{g} / \mathrm{mL}$ can safely prolong their dosing interval to every 3 weeks without loss of disease control.

Acknowledgements We are grateful to the research nurses and medical doctors of the Amsterdam Rheumatology and Immunology Center for seeing the patients and gathering the data, to A Marsman for her contribution to the inclusion of patients, and to J Ruwaard and E Vogelzang for their active participation in study 
discussions and their contribution in writing the manuscript. We also want to thank $T$ de Jong for handling and storing the blood samples.

Contributors MJA had full access to all of the data and takes responsibility for the integrity of the data and the accuracy of the data analysis. CLMK, MTN, MB and GJW were involved in the design of the study; MJA and CLMK acquired data; MJA analysed the data; and MJA, CLMK, MTN, TR, RFV, MB and GJW interpreted the data, prepared and edited the manuscript, and decided to submit the paper for publication.

Funding The study is partially funded by the Dutch Arthritis Foundation.

Disclaimer The funder of the study had no role in study design, data collection, data analysis, data interpretation, writing of the report or decision to submit the paper for publication.

Competing interests CLMK has received honoraria for lectures from Pfizer and MTN has received research funding or speaking/consultancy honoraria from AbbVie, Pfizer, Merck, Roche, BMS, UCB, Eli Lilly, Celgene and Janssen. RFV has received research support and grants from AbbVie, Amgen, BMS, GSK, Pfizer, Roche, UCB and honoraria for consultancy from AbbVie, Biotest, BMS, Celgene, Crescendo, GSK, Janssen, Lilly, Merck, Novartis, Pfizer, Roche, UCB and Vertex. TR has received honoraria for lectures from Pfizer, AbbVie and Regeneron, and a research grant from Genmab. MB has received consultancy for Pfizer, BMS, UCB and Teva. GJW has received research funding from Pfizer and honoraria for lectures and in advisory boards of Pfizer, UCB, BMS, AbbVie, Novartis and Biogen.

Ethics approval Slotervaart Hospital and Reade Medical Research Ethics Committee.

Provenance and peer review Not commissioned; externally peer reviewed.

(c) Article author(s) (or their employer(s) unless otherwise stated in the text of the article) 2018. All rights reserved. No commercial use is permitted unless otherwise expressly granted.

\section{REFERENCES}

1 Kievit W, Fransen J, Adang EM, et al. Long-term effectiveness and safety of TNFblocking agents in daily clinical practice: results from the Dutch Rheumatoid Arthritis Monitoring register. Rheumatology 2011:50:196-203.

2 Smolen JS, Landewé R, Bijlsma J, et al. EULAR recommendations for the management of rheumatoid arthritis with synthetic and biological disease-modifying antirheumatic drugs: 2016 update. Ann Rheum Dis 2017:76:960-77.

3 Ternant D, Bejan-Angoulvant T, Passot C, et al. Clinical pharmacokinetics and pharmacodynamics of monoclonal antibodies approved to treat rheumatoid arthritis. Clin Pharmacokinet 2015;54:1107-23.

4 Bartelds GM, Krieckaert CL, Nurmohamed MT, et al. Development of antidrug antibodies against adalimumab and association with disease activity and treatment failure during long-term follow-up. JAMA 2011;305:1460-8.

5 U.S. Food and Drug Administration. Clinical pharmacology and biopharmaceutics review(s). 2002 https://www.fda.gov/Drugs/DevelopmentApprovalProcess/H owDrugsareDevelopedandApproved/ApprovalApplications/TherapeuticBiologicApplica tions/ucm080610.htm (accessed 3 Mar 2017).
6 Pouw MF, Krieckaert CL, Nurmohamed MT, et al. Key findings towards optimising adalimumab treatment: the concentration-effect curve. Ann Rheum Dis 2015;74:513-8.

7 Chen DY, Chen YM, Hsieh TY, et al. Drug trough levels predict therapeutic responses to dose reduction of adalimumab for rheumatoid arthritis patients during 24 weeks of follow-up. Rheumatology 2016;55:143-8.

8 Bongartz T, Matteson EL, Montori VM, et al. Risk of serious infections and malignancies with anti-TNF antibody therapy in rheumatoid arthritis. JAMA 2006;296:2201-85.

9 Bejan-Angoulvant T, Ternant D, Daoued F, et al. Brief report: relationship between serum infliximab concentrations and risk of infections in patients treated for spondyloarthritis. Arthritis Rheumatol 2017;69:108-13.

10 Arnett FC, Edworthy SM, Bloch DA, et al. The American Rheumatism Association 1987 revised criteria for the classification of rheumatoid arthritis. Arthritis Rheum 1988;31:315-24

11 van der Heijde DM, van 't Hof MA, van Riel PL, et al. Judging disease activity in clinical practice in rheumatoid arthritis: first step in the development of a disease activity score. Ann Rheum Dis 1990;49:916-20.

12 van Herwaarden N, van der Maas A, Minten MJ, et al. Disease activity guided dose reduction and withdrawal of adalimumab or etanercept compared with usual care in rheumatoid arthritis: open label, randomised controlled, non-inferiority trial. BMJ 2015;350:h1389.

13 Fautrel B, Pham T, Alfaiate T, et al. Step-down strategy of spacing TNF-blocker injections for established rheumatoid arthritis in remission: results of the multicentre non-inferiority randomised open-label controlled trial (STRASS: Spacing of TNF-blocker injections in Rheumatoid ArthritiS Study). Ann Rheum Dis 2016:75:59-67.

14 Plasencia C, Wolbink G, Krieckaert CL, et al. Comparing a tapering strategy to the standard dosing regimen of TNF inhibitors in rheumatoid arthritis patients with low disease activity. Clin Exp Rheumatol 2016;34:655-62.

15 Smolen JS, Emery P, Fleischmann R, et al. Adjustment of therapy in rheumatoid arthritis on the basis of achievement of stable low disease activity with adalimumab plus methotrexate or methotrexate alone: the randomised controlled OPTIMA trial. Lancet 2014;383:321-32.

16 Tanaka Y, Hirata S, Kubo S, et al. Discontinuation of adalimumab after achieving remission in patients with established rheumatoid arthritis: 1-year outcome of the HONOR study. Ann Rheum Dis 2015;74:389-95.

17 van Herwaarden N, Bouman CA, van der Maas A, et al. Adalimumab and etanercept serum (anti)drug levels are not predictive for successful dose reduction or discontinuation in rheumatoid arthritis. Ann Rheum Dis 2015;74:2260-1.

18 Behrens F, Tony HP, Alten R, et al. Development and validation of a new disease activity score in 28 joints-based treatment response criterion for rheumatoid arthritis. Arthritis Care Res 2013;65:1608-16.

19 Menting SP, Coussens E, Pouw MF, et al. Developing a therapeutic range of adalimumab serum concentrations in management of psoriasis. JAMA Dermatol 2015;151:616-22.

20 Vogelzang EH, Kneepkens EL, Nurmohamed MT, et al. Anti-adalimumab antibodies and adalimumab concentrations in psoriatic arthritis; an association with disease activity at 28 and 52 weeks of follow-up. Ann Rheum Dis 2014;73:2178-82. 